\title{
A Case of Benign Chondroblastoma of Anterior Mandible and Review of Literature: A Very Rare Presentation
}

\author{
B. S. M. S. Siriwardena ${ }^{1}$, S. Shanmuganathan ${ }^{2}$, W. M. Tilakaratne ${ }^{1}$ \\ ${ }^{1}$ Department of Oral Pathology, Faculty of Dental Sciences, University of Peradeniya, Peradeniya, Sri Lanka \\ ${ }^{2}$ District General Hospital, Kalutara, Sri Lanka \\ Email: samadarani@yahoo.com, suresh1965@hotmail.com, wmtilak@pdn.ac.lk
}

Received 1 March 2015; accepted 14 March 2015; published 18 March 2015

Copyright $@ 2015$ by authors and Scientific Research Publishing Inc.

This work is licensed under the Creative Commons Attribution International License (CC BY).

http://creativecommons.org/licenses/by/4.0/

c) (i) Open Access

\begin{abstract}
Benign chondroblastoma of mandibular bone is an extremely rare tumor. Although 9 cases have been reported in the mandibular condyle in the English literature, to the best of our knowledge this is the first case originated from anterior mandible in a 31-year-old male.
\end{abstract}

\section{Keywords}

Mandible, Rare, Chondroblastoma

\section{Introduction}

Cartilage forming tumors are rare in the craniofacial region. Therefore, the risk of misdiagnosis can be significantly higher. The most common types of cartilage forming tumors in head and neck region are chondroma, both conventional and mesenchymal chondrosarcoma, chondroblastoma and chondromyxoid fibroma. Chondroblastoma is a benign tumor which occurs commonly in long bones of skeletally immature individuals. It is very rare in craniofacial bones accounting $6.4 \%$ of all chondroblastomas [1].

In 1931, Codman reported nine tumors containing giant cells with chondroid formation, which was named as "giant cell chondromatous tumor of the epiphysis" [2]. Later in 1942, Jaffe and Lichtenstein distinguished chondroblastoma from giant cell tumor, and coined the new term "benign chondroblastoma". Further they proposed that the possible origin of this tumor was from rests of the fetal chondroid remnants [3]. Chondroblastoma arising in the facial skeleton is a rare phenomenon and in the mandible it is much rarer. Although nine cases have been reported in the mandibular condyle so far in the English literature [4], we report the first case of benign chondroblastoma presented in the anterior mandible in a 31-year-old male. 


\section{Case Report}

A 31-year-old male presented to the Oral and maxillofacial Unit, Kalutara, Sri Lanka with a chief complaint of gradually increasing swelling over left anterior mandible with mobile lateral incisor for duration of $2-3$ months. Over a period of one year the patient had noticed that the canine had moved posteriorly. On examination there was a bony hard swelling about $1.5 \times 1.5 \mathrm{~cm}$ in relation to lower left lateral incisor (Figure 1). The patient was otherwise healthy and no history of trauma to the facial skeleton. Dental pantomograph revealed a well circumscribed unilocular radiolucent lesion with irregular margins. Resorption of roots was evident in central and lateral incisors as well as in canine of left side of the mandible (Figure 2). Lateral incisor showed marked resorption with grade III mobility. Fine needle aspiration cytology suggested a giant cell lesion. Serum calcium and alkaline phosphatase levels were within normal ranges. Subsequently the patient underwent exploratory surgery under general anesthesia (Figure 3(a)) and the biopsy was sent for histopathological investigation.

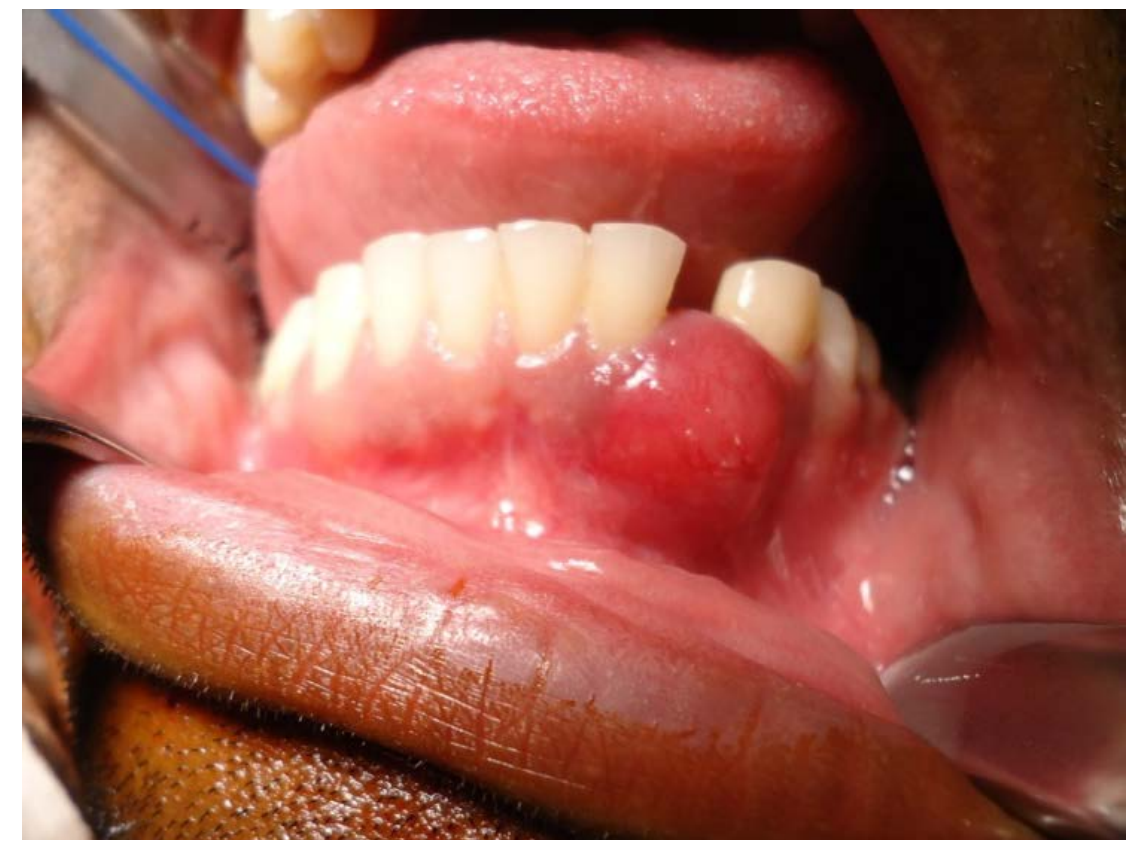

Figure 1. Clinical presentation.

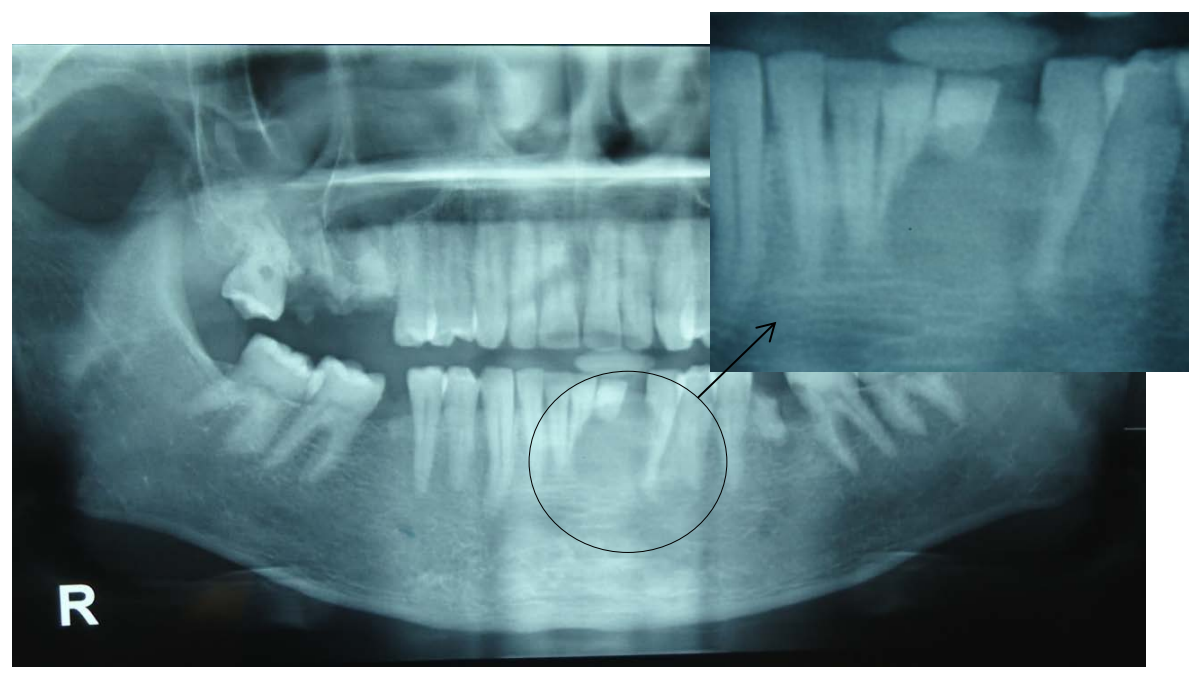

Figure 2. An oval shaped radiolucent lesion with an irregular margin. Resorption of roots is also present. 


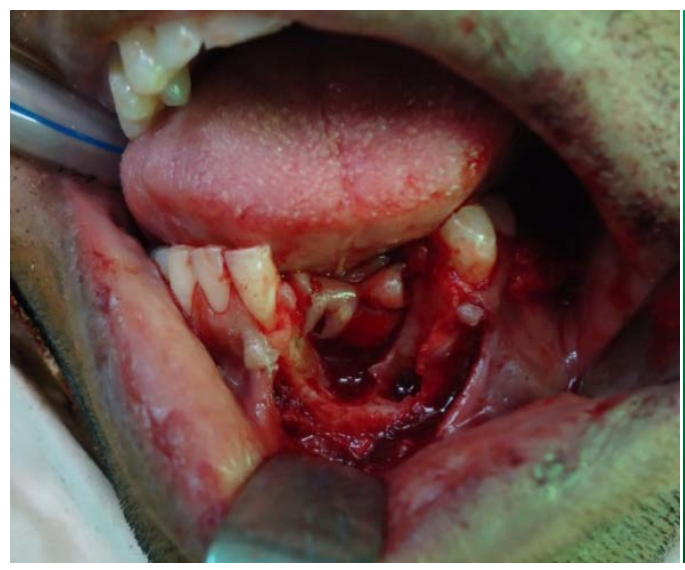

(a)

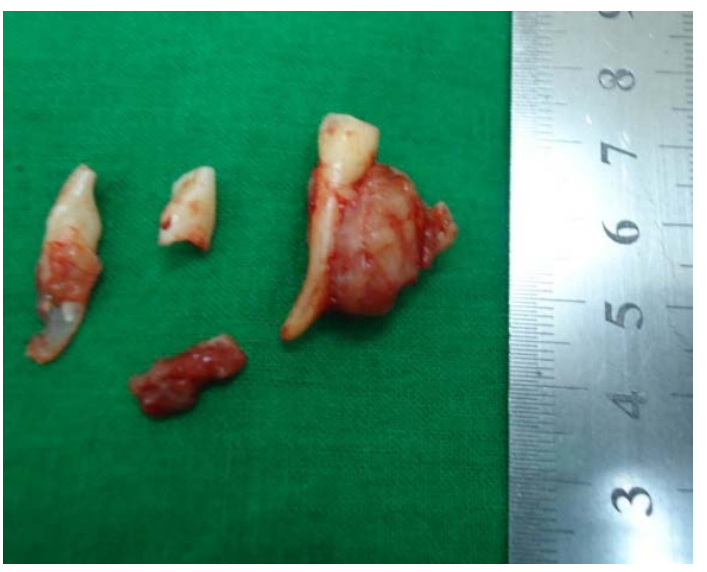

(b)

Figure 3. (a) After the exploratory surgery; (b) Macroscopic specimen.

Macroscopically the lesion was attached to the root of canine. There were incisors with irregularly resorbed roots (Figure 3(b)). Microscopically the tumour composed of mononuclear cells with well defined cell borders in a mucinous and chondroid matrix (Figure 4(a) and Figure 4(b)). A negative result for mucicarmine was used to exclude the presence of mucin. Majority of mononuclear cells were epitheliod in appearance with abundant eosinophilic cytoplasm and prominent nucleoli. The eosinophilic cells were arranged in to clusters and admixed with vacuolated cells. There were frequent multinucleate giant cells and no calcifications were found. Scattered occasional mitotic figures were observed throughout the lesion. Immunohistochemical stains for S-100 protein is negative in mononuclear cell population. Final diagnosis of benign chondroblastoma was made. No recurrence was observed five months in to the follow up.

\section{Discussion}

Chondroblastoma arising in craniofacial bones is rare and it accounts for $6.4 \%$ all chondroblastomas. Approximately $60 \%$ of all chondroblastomas occur in long bones mostly in the distal epiphysis of the femur [5]. Majority of craniofacial chondroblastomas involve the temporal bone. The next common site is mandiblular condyle. A few isolated cases have been reported in other bones of the skull [6]. The age ranges from 2 to 83 years and commonly affecting patients in the second decade in long bones. Mean age of occurrence in craniofacial skeleton tends to be 44 years which is older than in long bones [5] [6].

Clinically patients with chondroblastoma present to the clinician with localized pain. If the tumor involves temporal bone which is the most commonly affected, the frequent clinical symptoms are pain and loss of hearing [7]. Present case had mobility of teeth, pain and root resorption. Ideally, chondroblastoma radiographically appeared as a radiolucent lesion with a sclerotic margin. Radio-opacities, due to calcification, can clearly be seen in a CT and may or may not present in a plain radiograph [1] [7]. Present case also shows an oval shaped radiolucent lesion without radio-opacities.

Benign chondroblastoma in craniofacial skeleton is different histologically from those in long bones. Chondroid matrix is more bluish than pink. The matrix sometimes may be subtle or absent especially in tumors with prominent epitheliod cells [1]. Mitotic figures are usually present. However, frequent mitotic figures are not a common feature. Similar to chondroblastoma in other sites, the tumor is composed of mononuclear cells arranging in to nests or strands in a chondroid stroma. Frequent multinucleate giant cells are also observed. Epitheliod cells show eosinophilic cytoplasm and prominent nucleoli. The present tumor also showed that epitheliod cells arranged as clusters in a fibrous stroma and mononuclear cells were noted in a chondroid stroma. Although the characteristic feature of chondroblastoma is the presence of calcifications appeared as a chicken wire, $35 \%$ of the cases in the head and neck region shows the same [1]. Formation of secondary aneurysmal bone cyst can be seen up to one third of craniofacial lesions. Some cases show necrosis and permeation of tumor beyond the peripheral reactive rim of bone [1]. Though S-100 protein, EMA and Cytokeratin give some positivity in tumor cells, immunohistochemistry is not conclusive in diagnosing chondroblastoma.

The differential diagnosis should include giant cell tumor, chondomyxoid fibroma, chondrosarcoma, chon- 


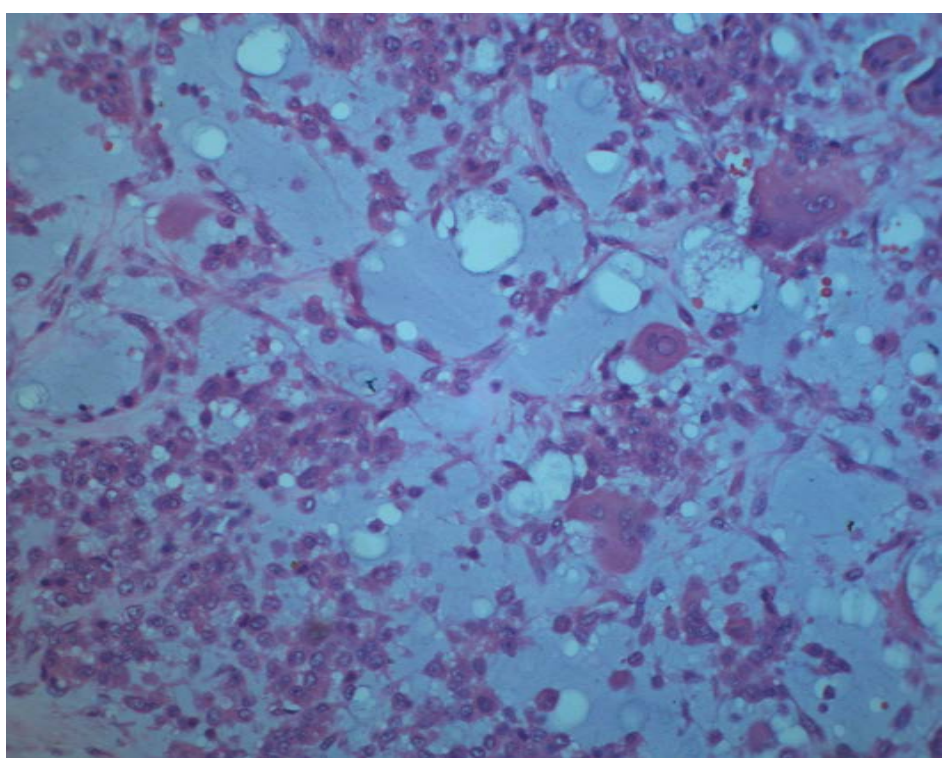

(a)

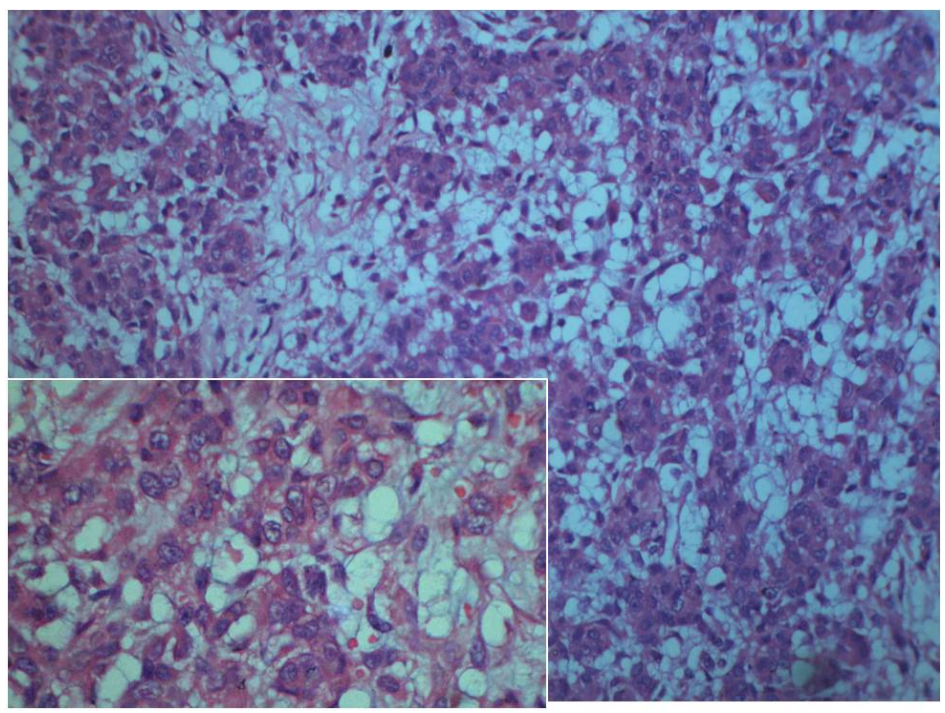

(b)

Figure 4. (a) Mononuclear cells in a chondroid stroma, scattered giant cells are present; (b) Epitheliod cells and vacuolated cells arranging in to clusters.

droblastic osteosarcoma and clear cell chondrosarcoma. Melanoma should be considered in tumors with epitheliod cells that contain brown pigments. The characteristic nuclear grooving is not present in mononuclear cells of giant cell tumor and chondroid matrix is absent. Although chondroblastoma and chondromyxoid fibroma show overlapping features, epitheliod cells are not seen in the latter. Melanoma can be excluded by Melan A immunohistochemically. Cytological atypia is noted in malignant tumors such as chondroblastic osteosarcoma and clear cell chondrosarcoma.

Curettage is the treatment of choice. It is necessary to remove the lesion completely without any trace of tumor in order to avoid recurrences. Reported recurrence rates vary from $6 \%$ to $15 \%$ [5] [6]. Periodic follow-up with radiological investigations is mandatory.

\section{Conclusion}

In conclusion, benign chondroblastoma is rare in the craniofacial skeleton. The mandible is an extremely rare site. 
Therefore, it is mandatory to exclude all other cartilage forming tumors before arriving at the final diagnosis of chondroblastoma.

\section{References}

[1] Inwards, C.Y. (2007) Update on Cartilage Forming Tumors of the Head and Neck. Head and Neck Pathology, 1, 67-74. http://dx.doi.org/10.1007/s12105-007-0015-4

[2] Codman, E.A. (1931) Epiphyseal Chondromatosus Giant Cell Tumors of the Upper End of the Humerus. Surg Gynecol Obstet, 52, 543-548.

[3] Jaffe, H.L. and Lichtenstein, L. (1942) Benign Chondroblastoma of Bone: A Reinterpretation of the So-Called Calcifying or Chondromatous Giant Cell Tumor. The American Journal of Pathology, 18, 969-991.

[4] Corrado, T., Massimo, R., Daniele, F., Salvatore, S., Nicoletta, Z. and Massimo, P. (2005) Chondroblastoma of the Mandibular Condyle: Case Report of an Extremely Uncommon Tumor. Oral Oncology Extra, 41, 132-136. http://dx.doi.org/10.1016/j.ooe.2005.03.001

[5] Arlen, M., Tollefsen, H.R., Huvos, A.G. and Marcove, R.C. (1970) Chondrosarcoma of the Head and Neck. The American Journal of Surgery, 120, 456-460. http://dx.doi.org/10.1016/S0002-9610(70)80006-X

[6] Bertoni, F., Unni, K.K., Beabout, J.W. and Sim, F.H. (1987) Chondroblastoma of the Skull and Facial Bones. American Journal of Clinical Pathology, 88, 1-9.

[7] Gaudet, E.G., Nuss, D.W., Johnson Jr., D.H. and Miranne Jr., L.S. (2004) Chondroblastoma of the Temporal Bone Involving the Temporomandibular Joint, Mandibular Condyle, and Middle Cranial Fossa: Case Report and Review of the Literature. Cranio, 22, 160-168. http://dx.doi.org/10.1179/crn.2004.021 